\title{
Are violent events responsible of a galaxy morphological loop?
}

\author{
Cecilia Scannapieco $^{1}$, A.V. Smith Castelli ${ }^{1,2}$ and P.B. Tissera ${ }^{1}$ \\ ${ }^{1}$ Instituto de Astronomía y Física del Espacio, Conicet - UBA, \\ CC 67 - Suc 28 (1428) Ciudad de Buenos Aires, Argentina \\ email: cecilia@iafe.uba.ar, patricia@iafe.uba.ar \\ ${ }^{2}$ Facultad de Ciencias Astronómicas y Geofísicas, Universidad Nacional de La Plata, \\ Paseo del Bosque s/n (1900) La Plata, Argentina \\ email: asmith@fcaglp.unlp.edu.ar
}

\begin{abstract}
We use cosmological SPH simulations to investigate the effects of mergers and interactions on the formation of the bulge and disc components of galactic systems. We find that secular evolution during mergers seems to be a key process in the formation of stable disc-bulge systems with observational counterparts and contributes to establish the fundamental relations observed in galaxies. Our findings suggest that the secular evolution phase couples the formation mechanisms of the bulge and disc components. According to our results, depending on the particular stability properties and merger parameters, violents events could drive a morphological loop in which the outcome could be a disc or a spheroid.
\end{abstract}

Keywords. methods: numerical, galaxies: interactions, galaxies: fundamental parameters

\section{Introduction}

The understanding of galaxy formation remains a primary goal in astrophysics. Within the current accepted scenario of structure formation, small galaxies are built up first and larger objects are assembled through the merging of less massive building blocks, in a hierarchical way. In this context, mergers and interactions play a crucial role modifying the properties of galaxies such as their mass distribution and star formation activity and affecting the subsequent evolution of the systems.

The formation of disc-like systems is still a matter of debate and several scenarios have been proposed in order to explain the growth of these two components and their interactions. The simplest scenarios currently accepted for the formation of the bulges are two: the first one establishes that the bulge and the disc are formed independently (Andredakis et al. 1995), and the second one assumes that the disc forms first and the bulge emerges from it as a consequence of gas inflow during a period of secular evolution (Courteau et al. 1996).

Numerical simulations are a powerful tool to tackle galaxy formation since they can account for the joint evolution of baryons and dark matter in a cosmological framework. We use Smooth Particle Hydrodynamical (SPH) simulations to investigate the effects of mergers and interactions on the determination of the structural properties of galaxies.

\section{Analysis and Discussion}

We analize galactic objects identified from numerical SPH simulations that take into account the gravitational and hydrodynamical evolution of the matter in Cold Dark Matter universes. We run two simulations with $N=64^{3}$ (S1) and $N=2 \times 80^{3}$ (S2) 
total number of particles and the following cosmological parameters: $\Lambda=0, h=0.5$, $\Omega_{b}=0.10$ (S1)and $\Lambda=0.7, h=0.7, \Omega_{b}=0.04$ (S2), where $\left(H=100 h \mathrm{~km} \mathrm{~s}^{-1} \mathrm{Mpc}^{-1}\right)$. The simulated boxes represent cubic volumes of $5 h^{-1}$ (S1) and $10 h^{-1}$ (S2) Mpc length.

We select galaxy-like objects (GLOs) at $z=0$ by using a density-contrast criterium and follow their evolution with look-back time, constructing their star formation and merger histories (see Scannapieco \& Tissera 2003 for details). With the aim at investigating the effects of mergers on the structural properties of the systems we perform bulge-disc decompositions to the projected mass surface density of the GLOs at $z=0$, and of the progenitors during merger events, assuming an exponential profile for the discs and the Sérsic law for the bulges. Galaxy-like objects identified in our simulations are found to have structural parameters similar to those of observed spiral galaxies (Sáiz et al. 2001).

Tissera et al. (2002) showed that during merger events, early gas inflows can be triggered depending on the stability properties of the systems. Those with shallower potential wells are more likely to suffer gas inflows since they can be more strongly perturbed from the incoming satellite. Secular evolution phases feed the bulge components which significantly contribute to the stability of the discs. The actual fusion of the baryonic cores increases the star formation activity which in turn affect the subsequent properties of the systems.

Understanding the effects of these two processes (i.e. secular evolution and fusion) on the growth of the disc and bulge components in galaxies can help us to enlighten the picture of galaxy formation. To this end we analize the evolution of the mass distribution of galaxy-like objects during merger events by studying the variation of their structural parameters. We find that secular evolution during the orbital decay phase produces the largest changes in the structural parameters of the systems: bulges get larger with respect to the discs and with more exponential profiles. These changes are found to be more important in unstable systems with shallower potential wells. Fusions introduce further modifications in the opposite direction although they are less important.

We also find that secular evolution phases contribute to establish the observed fundamental relations: after secular evolution, bulges tend to satisfy the observed Fundamental Plane and the disc components the Tully-Fisher relation. Fusions may add some dispersion but do not significantly change them (Smith Castelli et al. 2005). Departures from the Fundamental Relations could be indicating a lack of secular phases.

Our findings suggest that interactions play a fundamental role being able to transform discs into spheroids. Discs can also survive violent events, being either partially destroyed or just feeded by the colliding object. The stability properties of the initial system seem to be a fundamental point in the response of the disc to the incoming satellite. Other factors such as orbital parameters and relative masses of the colliding objects would also affect the determination of the structural properties of the outcome systems. The different possible histories of evolution of galactic systems in hierarchical universes could potentially explain the diversity of galaxies in our Universe.

\section{References}

Andredakis, Y.C., Peletier, R.F. \& Balcells, M. 1995, MNRAS 275, 874

Courteau, S., de Jong, R.S. \& Broeils, A.H. 1996, ApJ 457, L73

Sáiz, A., Domínguez-Tenreiro, R., Tissera, P.B. \& Courteau, S. 2001, MNRAS 325, 119

Scannapieco, C. \& Tissera, P.B. 2003, MNRAS 338, 880

Smith Castelli, A.V., Tissera, P.B. \& Scannapieco, C. 2005, MNRAS submitted

Tissera, P.B., Domínguez-Tenreiro, R., Scannapieco, C. \& Sáiz, A. 2002, MNRAS 333, 327 